\title{
Phonon Dispersion and Heat Capacity in $\beta$-Sheet Poly(L-alanine)
}

\author{
Radha Mohan MisRa, Vikal SAXENA, Poonam TANDon, ${ }^{\dagger}$ and Vishwambhar Dayal GuPTA
}

Physics Department, Lucknow University, Lucknow 226 007, India

(Received August 27, 2004; Accepted October 14, 2004; Published January 15, 2005)

\begin{abstract}
Earlier work on $\beta$-sheet poly(L-alanine) ( $\beta$ PLA) reported by Krishnan and Gupta suffers from several infirmities such as the replacement of the dynamic methyl group by a mass unit, which has resulted both in wrong assignments and incorrect profile of dispersion curves. In the present communication we report the phonon dispersion, density-of-states and heat capacity as a function of temperature in the range $200-400 \mathrm{~K}$, in $\beta$ PLA with all the atoms in the chemical repeat. Such studies have been already reported for the $\alpha$ PLA. The heat capacity is in good agreement with the measurements reported by Roles and Wunderlich. [DOI 10.1295/polymj.37.30]

KEY WORDS Poly(L-alanine) / $\beta$ Conformation / Phonon Dispersion / Heat Capacity / Densityof-States /
\end{abstract}

Synthetic homopolypeptides are a class of materials that form model compounds for the study of proteins. They are capable of taking up a variety of conformations. For example, poly( $\beta$-benzyl-L-aspartate) depending on the conditions of preparation, is capable of going into left-handed $\alpha$-helix, $\omega$-helix and $\beta$-pleated sheet. ${ }^{1}$ Similarly, glycine, proline and hydroxyproline are known to be helix breaker ${ }^{2}$ but their polymeric forms i.e., polyglycine II, poly(L-proline II) and poly(L-hydroxyproline) form left-handed 3-fold helix. Further these amino acids in collagen combine to form 3 -fold right-handed helix. Thus a study of the dynamical behaviour of polypeptides is very important in order to unravel the behaviour of proteins. Vibrational spectroscopy is an important tool for probing conformation through conformationally sensitive modes. The infrared absorption (IR), Raman spectra and inelastic neutron scattering (INS) from polymers in general and polypeptides in particular are very complex and can not be unraveled without the full knowledge of dispersion curves. One cannot appreciate without it the origin of both symmetry dependent and symmetry independent spectral feature. Analysis of infrared and Raman spectra calculated by the molecular dynamical method has also been recently reported. ${ }^{3}$ As in case of $\beta$ poly(L-valine $)^{4}$ ( $\beta$ PLV), dispersion curves also provide information on the extent of coupling along the chain together with an understanding of the dependence of the frequency of a given mode upon the sequence length of ordered conformation.

Next to polyglycine, poly(L-alanine) $\left(-\mathrm{NH}-\mathrm{C}_{\alpha} \mathrm{H}-\right.$ $\left.\mathrm{CH}_{3}-\mathrm{CO}-\right)_{n}$ is the simplest polypeptide. It exists in two crystalline forms differing in their chain conformations. One consists of $\alpha$-helices in a hexagonal close-packed arrangement with interchain bonds formed between $\mathrm{N}-\mathrm{H}$ and $\mathrm{C}=\mathrm{O}$ groups of alanine units three residue apart. ${ }^{5}$ The other form, known as a $\beta$ form, consists of antiparallel-pleated sheet having hydrogen bonds between $\mathrm{N}-\mathrm{H}$ and $\mathrm{C}=\mathrm{O}$ groups of adjacent polypeptide chains. ${ }^{6}$

In continuation to our work on the vibrational dynamics and phonon dispersion in polymers in general and polypeptides ${ }^{1,4,7-12}$ in particular, we report in the present communication phonon dispersion in $\beta$ PLA. Several workers ${ }^{12-14}$ have reported studies on this simple homopolypeptide. However, all these studies have one or the other infirmity. For example, Krishnan and Gupta ${ }^{12}$ have replaced $\mathrm{CH}_{3}$ group by an atom of mass 15 . This is a serious approximation because it hides all modes coupled with the dynamics of $\mathrm{CH}_{3}$ group. Moore and $\mathrm{Krimm}^{13}$ have reported normal modes analysis of $\beta$ PLA using only valance force field. Vandana and Krimm reported the phonon dispersion relation, density-of-states and heat capacity only for the $\alpha$ form of PLA. ${ }^{15}$ Such complete studies have not yet been reported in the literature for the $\beta$ form of PLA. Hence, it was felt that for a fuller understanding of the vibrational dynamics of $\beta$ PLA, a fresh look at it is necessary. In the present communication, we have critically reexamined the problem and removed all the infirmities as far as possible. We report here, phonon dispersion, density-of-states and heat capacity in $\beta$ PLA. Our calculations are based on UreyBradley force field, which in addition to valence force field accounts for the non-bonded interactions in the gem and cis configuration and tension terms. The normal mode frequencies obtained are found to be in better agreement with observed infrared and Raman bands than had been the case for the previous calculations. ${ }^{12}$

${ }^{\dagger}$ To whom correspondence should be addressed (E-mail: poonam_tandon@ @otmail.com). 


\section{THEORY}

\section{Calculation of Normal Mode Frequencies}

Normal mode calculation for a polymeric chain was carried out using Wilson's GF matrix method ${ }^{16}$ as modified by Higgs ${ }^{17}$ for an infinite polymeric chain. The vibrational secular equation to be solved is:

$$
|G(\delta) F(\delta)-\lambda(\delta) I|=0 \quad 0 \leq \delta \leq \pi
$$

where $\delta$ is the phase difference between the modes of adjacent chemical units, $G(\delta)$ is the inverse kinetic energy matrix and $F(\delta)$ is the force field matrix for a certain phase value. The frequencies $v_{i}$ in $\mathrm{cm}^{-1}$ are related to eigen values by $\lambda_{i}(\delta)=4 \pi^{2} c^{2} v_{i}^{2}(\delta)$.

A plot of $v_{i}(\delta) v s$. $\delta$ gives the dispersion curve for the $i$ th mode. The use of the type of force field is generally a matter of one's chemical experience and intuition. ${ }^{18}$ As stated earlier, in the present work we have used Urey-Bradley force field. ${ }^{19}$ Potential energy for this force field can be written as

$$
\begin{aligned}
V= & \sum_{m, j, k} K_{j, k}^{\prime} r_{j, k}^{(m)}\left(\Delta r_{j, k}^{(m)}\right)+K_{j, k}\left(\Delta r_{j, k}^{(m)}\right)^{2} / 2 \\
& +\sum_{m, i, j, k} H_{i, j, k}^{\prime} r_{i, j}^{(m)} r_{j, k}^{(m)}\left(\Delta \alpha_{i, j, k}^{(m)}\right) \\
& +H_{i, j, k} r_{j, k}^{(m)}\left(\Delta \alpha_{i, j, k}^{(m)}\right)^{2} / 2 \\
& +\sum_{m, i, j, k} F_{i, k}^{\prime} q_{i, k}^{(m)}\left(\Delta q_{i, k}^{(m)}\right)+F_{i, k}\left(\Delta q_{i, k}^{(m)}\right)^{2} / 2 \\
& +\sum_{j} K_{j}^{\tau}\left(\Delta \tau_{j}\right)^{2}+\sum_{j} K_{j}^{\omega}\left(\Delta \omega_{j}\right)^{2}
\end{aligned}
$$

where the symbols have their usual meaning. The primed quantities are introduced as internal tensions. Non-bonded interactions involve attraction and repulsion of atoms due to the overlap of their electron shells. These effects are usually expressed by the 6exp or 6-12 type potentials. The tension terms are assumed to be all zero.

Recently spectroscopically effective molecular mechanics model have been used for inter and intra molecular interactions consisting of charges, atomic dipoles and van der Waals interactions. ${ }^{20}$

The force constants, including those for the interaction of first and third non-bonded atoms, which give the "best fit", are given in the Table I and have been obtained by least squares fitting. In order to obtain the "best fit" with the observed IR, Raman, and INS spec$\operatorname{tra}^{14,21-24}$ the following procedure is adopted.

Force constants were initially transferred from the molecules (polyglycine I (PG I) ${ }^{10}$ and $\beta$ PLV $^{4}$ ) having similar groups placed in the similar environment.
Table I. Internal coordinates and force constants for $\beta$-sheet poly(L-alanine) (mydn/Å)

\begin{tabular}{lccc}
\hline $\begin{array}{c}\text { Internal } \\
\text { coordinates }\end{array}$ & $\begin{array}{c}\text { Force } \\
\text { constants }\end{array}$ & $\begin{array}{c}\text { Internal } \\
\text { coordinates }\end{array}$ & $\begin{array}{c}\text { Force } \\
\text { constants }\end{array}$ \\
\hline$v(\mathrm{C}=\mathrm{O})$ & 8.500 & $\varphi(\mathrm{O}=\mathrm{C}=\mathrm{N})$ & $0.580(0.120)$ \\
$v(\mathrm{C}=\mathrm{N})$ & 5.200 & $\varphi(\mathrm{C}=\mathrm{N}-\mathrm{H})$ & $0.330(0.570)$ \\
$v(\mathrm{~N}-\mathrm{H})$ & 5.170 & $\varphi\left(\mathrm{C}=\mathrm{N}-\mathrm{C}_{\alpha}\right)$ & $0.620(0.370)$ \\
$v\left(\mathrm{~N}-\mathrm{C}_{\alpha}\right)$ & 3.050 & $\varphi\left(\mathrm{H}-\mathrm{N}-\mathrm{C}_{\alpha}\right)$ & $0.320(0.570)$ \\
$v\left(\mathrm{C}_{\alpha}-\mathrm{H}_{\alpha}\right)$ & 3.940 & $\varphi\left(\mathrm{N}-\mathrm{C}_{\alpha}-\mathrm{H}_{\alpha}\right)$ & $0.386(0.810)$ \\
$v\left(\mathrm{C}_{\alpha}-\mathrm{C}_{\beta}\right)$ & 3.350 & $\varphi\left(\mathrm{N}-\mathrm{C}_{\alpha}-\mathrm{C}_{\beta}\right)$ & $0.130(0.500)$ \\
$v\left(\mathrm{C}_{\beta}-\mathrm{H}_{\beta}\right)$ & 4.350 & $\varphi\left(\mathrm{N}-\mathrm{C}_{\alpha}-\mathrm{C}\right)$ & $0.540(0.520)$ \\
$v\left(\mathrm{C}_{\alpha}-\mathrm{C}\right)$ & 2.750 & $\varphi\left(\mathrm{C}_{\beta}-\mathrm{C}_{\alpha}-\mathrm{C}\right)$ & $0.440(0.200)$ \\
$\omega(\mathrm{N}-\mathrm{H})$ & 0.146 & $\varphi\left(\mathrm{H}_{\alpha}-\mathrm{C}_{\alpha}-\mathrm{C}\right)$ & $0.480(0.200)$ \\
$\omega(\mathrm{C}=\mathrm{O})$ & 0.415 & $\varphi\left(\mathrm{H}_{\alpha}-\mathrm{C}_{\alpha}-\mathrm{C}_{\beta}\right)$ & $0.480(0.220)$ \\
$\tau\left(\mathrm{N}-\mathrm{C}_{\alpha}\right)$ & 0.031 & $\varphi\left(\mathrm{C}_{\alpha}-\mathrm{C}_{\beta}-\mathrm{H}_{\beta}\right)$ & $0.425(0.200)$ \\
$\tau\left(\mathrm{C}_{\alpha}-\mathrm{C}_{\beta}\right)$ & 0.013 & $\varphi\left(\mathrm{H}_{\beta}-\mathrm{C}_{\beta}-\mathrm{H}_{\beta}\right)$ & $0.400(0.270)$ \\
$\tau\left(\mathrm{C}_{\alpha}-\mathrm{C}\right)$ & 0.008 & $\varphi\left(\mathrm{C}_{\alpha}-\mathrm{C}=\mathrm{O}\right)$ & $0.520(0.800)$ \\
$\tau(\mathrm{C}=\mathrm{N})$ & 0.040 & $\varphi\left(\mathrm{C}_{\alpha}-\mathrm{C}=\mathrm{N}\right)$ & $0.500(0.600)$ \\
\multicolumn{5}{c}{ Off-diagonal interactions } \\
& $v(\mathrm{C}=\mathrm{N})-\varphi(\mathrm{O}=\mathrm{C}=\mathrm{N})$ & 0.560 \\
\hline
\end{tabular}

Note. $v, \varphi, \omega$ and $\tau$ denote stretch, angle bend, wag and torsion respectively. Non-bonded force constants are given in parenthesis.

Thus starting with the approximate $F$ matrix $F_{0}$ and observed frequencies $\lambda_{\text {obs }}$ (related through a constant), one can solve the secular matrix equation:

$$
G F_{0} L_{0}=L_{0} \lambda_{0}
$$

Let $\Delta \lambda_{i}=\lambda_{i \text { obs }}-\lambda_{i 0}$ in the above equation. It can be shown that in the direct order of approximation

$$
\Delta \lambda=J \Delta F
$$

where $J$ is computed from $L_{0}$. We wish to compute the corrections to $F_{0}$ so that the errors $\overline{\Delta \lambda}$ are minimized. We used the theory of least squares and calculate

$$
J^{\prime} P \overline{\Delta \lambda}=\left(J^{\prime} P J\right) \overline{\Delta F}
$$

where $P$ is the weighting matrix and $J^{\prime}$ is the transposition of $J$. The solution of this equation is obtained by inverting $J^{\prime} P J$ to give

$$
\overline{\Delta F}=\left(J^{\prime} P J\right)^{-1} J^{\prime} P \overline{\Delta \lambda}
$$

If the number of frequencies is greater than the number of $F$ matrix elements, the matrix $J^{\prime} P J$ should be non-singular and we obtain the corrections $\Delta F$, which will minimize the sum of the weighted squares of the residuals. This minimum sum provides the "best fit". If the corrections $\Delta F$ are fairly large, the linear relation between force constant and frequency term in the matrix equation (3) breaks down. In such a situation, further refinement using higher order terms in the Taylor's series expansion of $\Delta \lambda_{i}$ is needed. King et al. ${ }^{25}$ developed this procedure. 
Table II. Normal modes and their dispersion in $\beta$-sheet poly(L-alanine)

\begin{tabular}{|c|c|c|c|c|}
\hline \multirow{2}{*}{$\begin{array}{l}\text { Calc. } \\
\text { freq. }\end{array}$} & \multicolumn{2}{|c|}{ Obs. freq. } & \multirow{2}{*}{\multicolumn{2}{|c|}{$\begin{array}{l}\text { Assignment }(\delta=0) \\
\operatorname{PED}(\%)\end{array}$}} \\
\hline & IR & Raman & & \\
\hline 3242 & 3242 & - & $v(\mathrm{~N}-\mathrm{H})(100)$ & (Amide A) \\
\hline 2985 & 2985 & - & $v\left(\mathrm{C}_{\beta}-\mathrm{H}_{\beta}\right)(100)$ & \\
\hline 2927 & 2930 & 2933 & $v\left(\mathrm{C}_{\beta}-\mathrm{H}_{\beta}\right)(100)$ & \\
\hline 2927 & 2930 & 2933 & $v\left(\mathrm{C}_{\beta}-\mathrm{H}_{\beta}\right)(100)$ & \\
\hline 2871 & 2883 & 2871 & $v\left(\mathrm{C}_{\alpha}-\mathrm{H}_{\alpha}\right)(99)$ & \\
\hline 1671 & - & 1669 & $v(\mathrm{C}=\mathrm{O})(64)+v(\mathrm{C}-\mathrm{N})(17)+\varphi(\mathrm{C} \alpha-\mathrm{C}-\mathrm{N})(5)$ & (Amide I) \\
\hline 1536 & - & 1538 & $\varphi\left(\mathrm{H}-\mathrm{N}-\mathrm{C}_{\alpha}\right)(38)+\varphi(\mathrm{C}-\mathrm{N}-\mathrm{H})(34)+v(\mathrm{C}-\mathrm{N})(14)$ & (Amide II) \\
\hline 1446 & 1446 & 1451 & $\varphi\left(\mathrm{H}_{\beta}-\mathrm{C}_{\beta}-\mathrm{H}_{\beta}\right)(88)+\varphi\left(\mathrm{C}_{\alpha}-\mathrm{C}_{\beta}-\mathrm{H}_{\beta}\right)(5)$ & \\
\hline 1444 & 1446 & 1451 & $\varphi\left(\mathrm{H}_{\beta}-\mathrm{C}_{\beta}-\mathrm{H}_{\beta}\right)(94)$ & \\
\hline 1404 & 1400 & 1399 & $\begin{array}{l}\varphi\left(\mathrm{N}-\mathrm{C}_{\alpha}-\mathrm{H}_{\alpha}\right)(40)+\varphi\left(\mathrm{H}_{\beta}-\mathrm{C}_{\beta}-\mathrm{H}_{\beta}\right)(12)+v\left(\mathrm{C}_{\alpha}-\mathrm{C}_{\beta}\right)(9)+ \\
\varphi\left(\mathrm{C}_{\alpha}-\mathrm{C}_{\beta}-\mathrm{H}_{\beta}\right)(9)+\varphi\left(\mathrm{H}_{\alpha}-\mathrm{C}_{\alpha}-\mathrm{C}_{\beta}\right)(8)+v\left(\mathrm{~N}-\mathrm{C}_{\alpha}\right)(8)\end{array}$ & \\
\hline 1392 & 1380 & - & $\begin{array}{l}\varphi\left(\mathrm{H}_{\beta}-\mathrm{C}_{\beta}-\mathrm{H}_{\beta}\right)(27)+\varphi\left(\mathrm{C}_{\alpha}-\mathrm{C}_{\beta}-\mathrm{H}_{\beta}\right)(27)+\varphi\left(\mathrm{N}-\mathrm{C}_{\alpha}-\mathrm{H}_{\alpha}\right)(17)+ \\
v\left(\mathrm{C}_{\alpha}-\mathrm{C}_{\beta}\right)(10)+\varphi\left(\mathrm{H}_{\alpha}-\mathrm{C}_{\alpha}-\mathrm{C}\right)(10)\end{array}$ & \\
\hline 1330 & 1332 & 1335 & $\begin{array}{l}\varphi\left(\mathrm{H}_{\alpha}-\mathrm{C}_{\alpha}-\mathrm{C}_{\beta}\right)(26)+\varphi\left(\mathrm{H}_{\alpha}-\mathrm{C}_{\alpha}-\mathrm{C}\right)(19)+v\left(\mathrm{C}_{\alpha}-\mathrm{C}\right)(14)+ \\
v(\mathrm{C}-\mathrm{N})(10)+\varphi\left(\mathrm{C}_{\alpha}-\mathrm{C}_{\beta}-\mathrm{H}_{\beta}\right)(9)+\varphi\left(\mathrm{H}_{\beta}-\mathrm{C}_{\beta}-\mathrm{H}_{\beta}\right)(7)\end{array}$ & \\
\hline 1224 & 1223 & 1221 & $\begin{array}{l}v(\mathrm{C}-\mathrm{N})(34)+\varphi\left(\mathrm{H}_{\alpha}-\mathrm{C}_{\alpha}-\mathrm{C}\right)(12)+v\left(\mathrm{~N}-\mathrm{C}_{\alpha}\right)(10)+v\left(\mathrm{C}_{\alpha}-\mathrm{C}\right)(8)+ \\
\varphi\left(\mathrm{H}_{\alpha}-\mathrm{C}_{\alpha}-\mathrm{C}_{\beta}\right)(7)+\varphi\left(\mathrm{H}-\mathrm{N}-\mathrm{C}_{\alpha}\right)(7)+v(\mathrm{C}=\mathrm{O})(6)+ \\
\varphi(\mathrm{O}=\mathrm{C}-\mathrm{N})(6)\end{array}$ & (Amide III) \\
\hline 1173 & 1167 & 1165 & $v\left(\mathrm{~N}-\mathrm{C}_{\alpha}\right)(44)+v\left(\mathrm{C}_{\alpha}-\mathrm{C}\right)(17)+\varphi\left(\mathrm{C}_{\alpha}-\mathrm{C}_{\beta}-\mathrm{H}_{\beta}\right)(7)+\varphi\left(\mathrm{C}_{\alpha}-\mathrm{C}=\mathrm{O}\right)(5)$ & \\
\hline 1060 & 1050 & 1069 & $\begin{array}{l}v\left(\mathrm{C}_{\alpha}-\mathrm{C}\right)(17)+v\left(\mathrm{C}_{\alpha}-\mathrm{C}_{\beta}\right)(16)+v(\mathrm{C}=\mathrm{O})(12)+\varphi\left(\mathrm{H}_{\alpha}-\mathrm{C}_{\alpha}-\mathrm{C}\right)(8)+ \\
\varphi\left(\mathrm{H}_{\alpha}-\mathrm{C}_{\alpha}-\mathrm{C}_{\beta}\right)(8)+\varphi\left(\mathrm{C}_{\alpha}-\mathrm{C}_{\beta}-\mathrm{H}_{\beta}\right)(8)+v(\mathrm{C}-\mathrm{N})(6)+\varphi\left(\mathrm{C}-\mathrm{N}-\mathrm{C}_{\alpha}\right)(6)\end{array}$ & \\
\hline 981 & 967 & 967 & $\varphi\left(\mathrm{C}_{\alpha}-\mathrm{C}_{\beta}-\mathrm{H}_{\beta}\right)(58)+v\left(\mathrm{C}_{\alpha}-\mathrm{C}_{\beta}\right)(17)$ & \\
\hline 896 & 906 & 909 & $\varphi\left(\mathrm{C}_{\alpha}-\mathrm{C}_{\beta}-\mathrm{H}_{\beta}\right)(77)+v\left(\mathrm{~N}-\mathrm{C}_{\alpha}\right)(8)+v\left(\mathrm{C}_{\alpha}-\mathrm{C}\right)(8)$ & \\
\hline 891 & - & - & $\begin{array}{l}v\left(\mathrm{C}_{\alpha}-\mathrm{C}_{\beta}\right)(29)+\varphi\left(\mathrm{C}_{\alpha}-\mathrm{C}_{\beta}-\mathrm{H}_{\beta}\right)(27)+\varphi(\mathrm{O}=\mathrm{C}-\mathrm{N})(13)+ \\
v\left(\mathrm{~N}-\mathrm{C}_{\alpha}\right)(6)+\varphi\left(\mathrm{C}-\mathrm{N}-\mathrm{C}_{\alpha}\right)(6)\end{array}$ & \\
\hline 775 & 771 & 775 & $\omega(\mathrm{N}-\mathrm{H})(37)+\omega(\mathrm{C}=\mathrm{O})(26)+\varphi\left(\mathrm{N}-\mathrm{C}_{\alpha}-\mathrm{C}\right)(9)+\varphi\left(\mathrm{C}_{\alpha}-\mathrm{C}_{\beta}-\mathrm{H}_{\beta}\right)(8)$ & \\
\hline 631 & 622 & - & $\begin{array}{l}\omega(\mathrm{N}-\mathrm{H})(30)+\tau(\mathrm{C}-\mathrm{N})(18)+\tau\left(\mathrm{N}-\mathrm{C}_{\alpha}\right)(13)+\omega(\mathrm{C}=\mathrm{O})(13)+ \\
\varphi\left(\mathrm{N}-\mathrm{C}_{\alpha}-\mathrm{C}\right)(5)\end{array}$ & (Amide V) \\
\hline 528 & 528 & 531 & $\begin{array}{l}\varphi\left(\mathrm{C}_{\alpha}-\mathrm{C}=\mathrm{O}\right)(29)+\varphi(\mathrm{O}=\mathrm{C}-\mathrm{N})(24)+\omega(\mathrm{C}=\mathrm{O})(20)+ \\
v\left(\mathrm{C}_{\alpha}-\mathrm{C}\right)(11)+v\left(\mathrm{C}_{\alpha}-\mathrm{C}_{\beta}\right)(7)\end{array}$ & (Amide IV + Amide VI) \\
\hline 392 & 372 & 375 & $\begin{array}{l}\omega(\mathrm{C}=\mathrm{O})(21)+\varphi\left(\mathrm{N}-\mathrm{C}_{\alpha}-\mathrm{C}_{\beta}\right)(13)+\varphi\left(\mathrm{C}_{\alpha}-\mathrm{C}=\mathrm{O}\right)(10)+ \\
\varphi\left(\mathrm{H}_{\alpha}-\mathrm{C}_{\alpha}-\mathrm{C}_{\beta}\right)(8)+\varphi\left(\mathrm{H}_{\alpha}-\mathrm{C}_{\alpha}-\mathrm{C}\right)(7)+\varphi\left(\mathrm{C}_{\alpha}-\mathrm{C}-\mathrm{N}\right)(7)+ \\
\varphi\left(\mathrm{C}_{\beta}-\mathrm{C}_{\alpha}-\mathrm{C}\right)(6)+v\left(\mathrm{~N}-\mathrm{C}_{\alpha}\right)(6)\end{array}$ & \\
\hline 326 & 326 & 332 & $\begin{array}{l}\varphi\left(\mathrm{N}-\mathrm{C}_{\alpha}-\mathrm{C}_{\beta}\right)(32)+\varphi\left(\mathrm{C}_{\beta}-\mathrm{C}_{\alpha}-\mathrm{C}\right)(31)+\varphi\left(\mathrm{C}_{\alpha}-\mathrm{C}-\mathrm{N}\right)(9)+ \\
\varphi\left(\mathrm{C}-\mathrm{N}-\mathrm{C}_{\alpha}\right)(9)+\varphi(\mathrm{O}=\mathrm{C}-\mathrm{N})(7)\end{array}$ & \\
\hline 259 & - & 266 & $\tau\left(\mathrm{C}_{\alpha}-\mathrm{C}_{\beta}\right)(88)$ & \\
\hline 187 & - & 185 & $\begin{array}{l}\tau\left(\mathrm{N}-\mathrm{C}_{\alpha}\right)(33)+\omega(\mathrm{C}=\mathrm{O})(12)+\omega(\mathrm{N}-\mathrm{H})(12)+\varphi\left(\mathrm{N}-\mathrm{C}_{\alpha}-\mathrm{C}\right)(11)+ \\
\tau\left(\mathrm{C}_{\alpha}-\mathrm{C}_{\beta}\right)(8)+\tau(\mathrm{C}-\mathrm{N})(6)\end{array}$ & \\
\hline 182 & - & 185 & $\begin{array}{l}\varphi\left(\mathrm{C}_{\beta}-\mathrm{C}_{\alpha}-\mathrm{C}\right)(26)+\varphi\left(\mathrm{N}-\mathrm{C}_{\alpha}-\mathrm{C}\right)(24)+\varphi\left(\mathrm{C}_{\alpha}-\mathrm{C}-\mathrm{N}\right)(15)+ \\
\varphi\left(\mathrm{C}_{\alpha}-\mathrm{C}=\mathrm{O}\right)(10)+\varphi\left(\mathrm{N}-\mathrm{C}_{\alpha}-\mathrm{C}_{\beta}\right)(6)\end{array}$ & \\
\hline 137 & - & $135-$ & $\begin{array}{l}\varphi\left(\mathrm{C}-\mathrm{N}-\mathrm{C}_{\alpha}\right)(33)+\varphi\left(\mathrm{N}-\mathrm{C}_{\alpha}-\mathrm{C}_{\beta}\right)(23)+\varphi(\mathrm{O}=\mathrm{C}-\mathrm{N})(9)+ \\
\varphi\left(\mathrm{C}_{\alpha}-\mathrm{C}-\mathrm{N}\right)(8)+\varphi(\mathrm{C}-\mathrm{N}-\mathrm{H})(6)+\varphi\left(\mathrm{H}-\mathrm{N}-\mathrm{C}_{\alpha}\right)(5)\end{array}$ & \\
\hline 83 & - & 91 & $\tau(\mathrm{C}-\mathrm{N})(43)+\tau\left(\mathrm{C}_{\alpha}-\mathrm{C}\right)(24)+\omega(\mathrm{N}-\mathrm{H})(12)+\varphi\left(\mathrm{N}-\mathrm{C}_{\alpha}-\mathrm{C}\right)(6)$ & (Amide VII) \\
\hline
\end{tabular}

\section{Calculation of Specific Heat}

Dispersion curves can be used to calculate the specific heat of a polymeric system. For a one-dimensional system the density-of-state function or the frequency distribution function, which expresses the way energy is distributed among the various branches of normal modes in the crystal, is calculated from the relation

$$
\left.g(v)=\Sigma\left(\partial v_{j} / \partial \delta\right)^{-1}\right]_{v j(\delta)=v j}
$$

The sum is over all the branches $j$. Considering a solid as an assembly of harmonic oscillators, the frequency distribution $g(v)$ is equivalent to a partition function. The constant volume heat capacity can be calculated using Debye's relation

$$
\begin{aligned}
C_{v}= & \Sigma g\left(v_{j}\right) K N_{A}\left(h v_{j} / K T\right)^{2} \\
& \left.\times\left[\exp \left(h v_{j} / K T\right) /\left\{\exp \left(h v_{j} / K T\right)-1\right)\right\}^{2}\right]
\end{aligned}
$$

with 


\begin{tabular}{|c|c|c|c|c|}
\hline \multirow{2}{*}{$\begin{array}{l}\text { Calc. } \\
\text { freq. }\end{array}$} & \multicolumn{2}{|c|}{ Obs. freq. } & \multirow{2}{*}{\multicolumn{2}{|c|}{$\begin{array}{l}\text { Assignment }(\delta=\pi) \\
\operatorname{PED}(\%)\end{array}$}} \\
\hline & IR & Raman & & \\
\hline 3242 & 3242 & - & $v(\mathrm{~N}-\mathrm{H})(100)$ & (Amide A) \\
\hline 2985 & 2985 & - & $v\left(\mathrm{C}_{\beta}-\mathrm{H}_{\beta}\right)(100)$ & \\
\hline 2927 & 2930 & 2933 & $v\left(\mathrm{C}_{\beta}-\mathrm{H}_{\beta}\right)(100)$ & \\
\hline 2927 & 2930 & 2933 & $v\left(\mathrm{C}_{\beta}-\mathrm{H}_{\beta}\right)(100)$ & \\
\hline 2871 & 2883 & 2871 & $v\left(\mathrm{C}_{\alpha}-\mathrm{H}_{\alpha}\right)(99)$ & \\
\hline 1663 & - & 1669 & $v(\mathrm{C}=\mathrm{O})(68)+v(\mathrm{C}-\mathrm{N})(14)$ & (Amide I) \\
\hline 1536 & - & 1538 & $\varphi\left(\mathrm{H}-\mathrm{N}-\mathrm{C}_{\alpha}\right)(36)+\varphi(\mathrm{C}-\mathrm{N}-\mathrm{H})(34)+v(\mathrm{C}-\mathrm{N})(17)$ & (Amide II) \\
\hline 1446 & 1446 & 1451 & $\varphi\left(\mathrm{H}_{\beta}-\mathrm{C}_{\beta}-\mathrm{H}_{\beta}\right)(87)+\varphi\left(\mathrm{C}_{\alpha}-\mathrm{C}_{\beta}-\mathrm{H}_{\beta}\right)(5)$ & \\
\hline 1444 & 1446 & 1451 & $\varphi\left(\mathrm{H}_{\beta}-\mathrm{C}_{\beta}-\mathrm{H}_{\beta}\right)(94)$ & \\
\hline 1406 & 1400 & 1399 & $\begin{array}{l}\varphi\left(\mathrm{N}-\mathrm{C}_{\alpha}-\mathrm{H}_{\alpha}\right)(42)+\varphi\left(\mathrm{H}_{\beta}-\mathrm{C}_{\beta}-\mathrm{H}_{\beta}\right)(10)+\varphi\left(\mathrm{H}_{\alpha}-\mathrm{C}_{\alpha}-\mathrm{C}_{\beta}\right)(8)+ \\
v\left(\mathrm{C}_{\alpha}-\mathrm{C}_{\beta}\right)(7)+\varphi\left(\mathrm{C}_{\alpha}-\mathrm{C}_{\beta}-\mathrm{H}_{\beta}\right)(7)+v\left(\mathrm{~N}-\mathrm{C}_{\alpha}\right)(6)+\varphi\left(\mathrm{H}_{\alpha}-\mathrm{C}_{\alpha}-\mathrm{C}\right)(5)\end{array}$ & \\
\hline 1389 & 1380 & - & $\begin{array}{l}\varphi\left(\mathrm{C}_{\alpha}-\mathrm{C}_{\beta}-\mathrm{H}_{\beta}\right)(32)+\varphi\left(\mathrm{H}_{\beta}-\mathrm{C}_{\beta}-\mathrm{H}_{\beta}\right)(32)+\varphi\left(\mathrm{N}-\mathrm{C}_{\alpha}-\mathrm{H}_{\alpha}\right)(14)+ \\
v\left(\mathrm{C}_{\alpha}-\mathrm{C}_{\beta}\right)(11)+\varphi\left(\mathrm{H}_{\alpha}-\mathrm{C}_{\alpha}-\mathrm{C}\right)(7)\end{array}$ & \\
\hline 1312 & 1308 & 1311 & $\begin{array}{l}\varphi\left(\mathrm{H}_{\alpha}-\mathrm{C}_{\alpha}-\mathrm{C}_{\beta}\right)(34)+\varphi\left(\mathrm{H}_{\alpha}-\mathrm{C}_{\alpha}-\mathrm{C}\right)(32)+\varphi\left(\mathrm{C}_{\alpha}-\mathrm{C}_{\beta}-\mathrm{H}_{\beta}\right)(6)+ \\
v\left(\mathrm{C}_{\alpha}-\mathrm{C}_{\beta}\right)(6)\end{array}$ & \\
\hline 1236 & 1243 & 1241 & $\begin{array}{l}v(\mathrm{C}-\mathrm{N})(29)+v\left(\mathrm{C}_{\alpha}-\mathrm{C}\right)(21)+v\left(\mathrm{~N}-\mathrm{C}_{\alpha}\right)(15)+\varphi(\mathrm{O}=\mathrm{C}-\mathrm{N})(7)+ \\
\varphi\left(\mathrm{H}-\mathrm{N}-\mathrm{C}_{\alpha}\right)(6)+v(\mathrm{C}=\mathrm{O})(5)\end{array}$ & (Amide III) \\
\hline 1092 & - & 1096 & $\begin{array}{l}v\left(\mathrm{~N}-\mathrm{C}_{\alpha}\right)(32)+v\left(\mathrm{C}_{\alpha}-\mathrm{C}_{\beta}\right)(14)+v(\mathrm{C}-\mathrm{N})(13)+v\left(\mathrm{C}_{\alpha}-\mathrm{C}\right)(9)+ \\
\varphi\left(\mathrm{H}_{\alpha}-\mathrm{C}_{\alpha}-\mathrm{C}\right)(6)+\varphi\left(\mathrm{N}-\mathrm{C}_{\alpha}-\mathrm{C}\right)(5)+\varphi\left(\mathrm{C}_{\alpha}-\mathrm{C}_{\beta}-\mathrm{H}_{\beta}\right)(5)\end{array}$ & \\
\hline 1005 & 1017 & - & $\begin{array}{l}\varphi\left(\mathrm{C}_{\alpha}-\mathrm{C}_{\beta}-\mathrm{H}_{\beta}\right)(27)+v\left(\mathrm{C}_{\alpha}-\mathrm{C}_{\beta}\right)(27)+v\left(\mathrm{C}_{\alpha}-\mathrm{C}\right)(9)+v(\mathrm{C}-\mathrm{N})(8)+ \\
v\left(\mathrm{~N}-\mathrm{C}_{\alpha}\right)(7)+\varphi\left(\mathrm{H}_{\alpha}-\mathrm{C}_{\alpha}-\mathrm{C}_{\beta}\right)(6)\end{array}$ & \\
\hline 991 & - & - & $\varphi\left(\mathrm{C}_{\alpha}-\mathrm{C}_{\beta}-\mathrm{H}_{\beta}\right)(47)+v\left(\mathrm{C}_{\alpha}-\mathrm{C}\right)(12)+v\left(\mathrm{C}_{\alpha}-\mathrm{C}_{\beta}\right)(8)+v(\mathrm{C}-\mathrm{N})(5)$ & \\
\hline 920 & 926 & - & $\varphi\left(\mathrm{C}_{\alpha}-\mathrm{C}_{\beta}-\mathrm{H}_{\beta}\right)(53)+\varphi(\mathrm{O}=\mathrm{C}-\mathrm{N})(12)+v\left(\mathrm{C}_{\alpha}-\mathrm{C}\right)(8)+\varphi\left(\mathrm{C}-\mathrm{N}-\mathrm{C}_{\alpha}\right)(6)$ & \\
\hline 870 & - & - & $\Phi\left(\mathrm{C}_{\alpha}-\mathrm{C}_{\beta}-\mathrm{H}_{\beta}\right)(43)+v\left(\mathrm{~N}-\mathrm{C}_{\alpha}\right)(23)+v\left(\mathrm{C}_{\alpha}-\mathrm{C}\right)(10)+v\left(\mathrm{C}_{\alpha}-\mathrm{C}_{\beta}\right)(8)$ & \\
\hline 703 & 709 & - & $\omega(\mathrm{N}-\mathrm{H})(64)+\tau(\mathrm{C}-\mathrm{N})(10)+\omega(\mathrm{C}=\mathrm{O})(7)$ & (Amide $\mathrm{V}$ ) \\
\hline 699 & - & 698 & $\begin{array}{l}\varphi(\mathrm{O}=\mathrm{C}-\mathrm{N})(13)+v\left(\mathrm{C}_{\alpha}-\mathrm{C}\right)(11)+\omega(\mathrm{C}=\mathrm{O})(10)+\varphi\left(\mathrm{C}_{\alpha}-\mathrm{C}_{\beta}-\mathrm{H}_{\beta}\right)(9)+v(\mathrm{~N}- \\
\left.\mathrm{C}_{\alpha}\right)(8)+\varphi\left(\mathrm{N}-\mathrm{C}_{\alpha}-\mathrm{C}\right)(7)+\varphi\left(\mathrm{C}-\mathrm{N}-\mathrm{C}_{\alpha}\right)(7)+\omega(\mathrm{N}-\mathrm{H})(7)+v(\mathrm{C}-\mathrm{N})(5)\end{array}$ & (Amide IV) \\
\hline 646 & 657 & - & $\omega(\mathrm{C}=\mathrm{O})(50)+\tau\left(\mathrm{N}-\mathrm{C}_{\alpha}\right)(12)+\varphi\left(\mathrm{N}-\mathrm{C}_{\alpha}-\mathrm{C}\right)(8)$ & (Amide VI) \\
\hline 507 & - & - & $\begin{array}{l}\varphi\left(\mathrm{C}_{\alpha}-\mathrm{C}-\mathrm{N}\right)(22)+\varphi\left(\mathrm{C}_{\alpha}-\mathrm{C}=\mathrm{O}\right)(19)+\varphi\left(\mathrm{N}-\mathrm{C}_{\alpha}-\mathrm{C}\right)(18)+\tau\left(\mathrm{N}-\mathrm{C}_{\alpha}\right)(7)+ \\
v\left(\mathrm{C}_{\alpha}-\mathrm{C}_{\beta}\right)(6)+\omega(\mathrm{C}=\mathrm{O})(6)+\varphi\left(\mathrm{C}_{\beta}-\mathrm{C}_{\alpha}-\mathrm{C}\right)(5)\end{array}$ & \\
\hline 438 & 432 & 437 & $\begin{array}{l}\varphi\left(\mathrm{H}_{\alpha}-\mathrm{C}_{\alpha}-\mathrm{C}_{\beta}\right)(14)+\varphi\left(\mathrm{N}-\mathrm{C}_{\alpha}-\mathrm{C}_{\beta}\right)(13)+\varphi\left(\mathrm{C}_{\beta}-\mathrm{C}_{\alpha}-\mathrm{C}\right)(12)+\tau(\mathrm{C}-\mathrm{N})(10)+ \\
\tau\left(\mathrm{N}-\mathrm{C}_{\alpha}\right)(8)+\varphi\left(\mathrm{H}_{\alpha}-\mathrm{C}_{\alpha}-\mathrm{C}\right)(7)+\varphi\left(\mathrm{C}_{\alpha}-\mathrm{C}-\mathrm{N}\right)(7)+\varphi\left(\mathrm{N}-\mathrm{C}_{\alpha}-\mathrm{H}_{\alpha}\right)(6)\end{array}$ & \\
\hline 299 & - & 300 & $\begin{array}{l}\varphi(\mathrm{O}=\mathrm{C}-\mathrm{N})(26)+\varphi\left(\mathrm{N}-\mathrm{C}_{\alpha}-\mathrm{C}\right)(19)+\varphi\left(\mathrm{C}_{\alpha}-\mathrm{C}=\mathrm{O}\right)(19)+\varphi\left(\mathrm{C}-\mathrm{N}-\mathrm{C}_{\alpha}\right)(16)+ \\
\varphi\left(\mathrm{N}-\mathrm{C}_{\alpha}-\mathrm{C}_{\beta}\right)(7)\end{array}$ & \\
\hline 247 & 240 & 235 & $\tau\left(\mathrm{C}_{\alpha}-\mathrm{C}_{\beta}\right)(96)$ & \\
\hline 182 & - & 185 & $\varphi\left(\mathrm{C}_{\beta}-\mathrm{C}_{\alpha}-\mathrm{C}\right)(46)+\varphi\left(\mathrm{N}-\mathrm{C}_{\alpha}-\mathrm{C}_{\beta}\right)(36)+\varphi\left(\mathrm{C}_{\alpha}-\mathrm{C}-\mathrm{N}\right)(5)$ & \\
\hline 117 & 122 & - & $\tau\left(\mathrm{C}_{\alpha}-\mathrm{C}\right)(42)+\tau\left(\mathrm{N}-\mathrm{C}_{\alpha}\right)(24)+\omega(\mathrm{C}=\mathrm{O})(5)$ & \\
\hline 101 & 122 & - & $\begin{array}{l}\tau\left(\mathrm{N}-\mathrm{C}_{\alpha}\right)(29)+\tau(\mathrm{C}-\mathrm{N})(25)+\varphi\left(\mathrm{C}-\mathrm{N}-\mathrm{C}_{\alpha}\right)(10)+\varphi\left(\mathrm{N}-\mathrm{C}_{\alpha}-\mathrm{C}\right)(8)+ \\
\varphi\left(\mathrm{N}-\mathrm{C}_{\alpha}-\mathrm{C}_{\beta}\right)(8)\end{array}$ & (Amide VII) \\
\hline
\end{tabular}

All frequencies are in $\mathrm{cm}^{-1}$

$$
\int g\left(v_{i}\right) \mathrm{d} v_{i}=1
$$

The constant-volume heat capacity $C_{\mathrm{v}}$, given by above equation, can be converted into constant-pressure heat capacity $C_{\mathrm{p}}$ using the Nernst-Lindemann approximation. ${ }^{26}$

$$
C_{\mathrm{p}}-C_{\mathrm{v}}=3 R A_{0}\left(C_{\mathrm{p}}^{2} T / C_{\mathrm{v}} T_{\mathrm{m}}^{0}\right)
$$

Where $A_{0}$ is a constant often of a universal value $\left[3.90 \times 10^{-3}(\mathrm{~K} \mathrm{~mol}) / \mathrm{J}\right]$ and $T_{\mathrm{m}}^{0}$ is the estimated equilibrium melting temperature, which is taken to be $573 \mathrm{~K}^{27}$

\section{RESULTS AND DISCUSSION}

Figure 1 represents the chemical repeat unit of PLA. It has 10 atoms per repeat residue that give rise to 30 dispersion curves. The vibrational frequencies were calculated for values of $\delta$ varying from 0 to $\pi$ in steps of $0.05 \pi$. The optically active modes are those for which $\delta=0$ or $\delta=\pi$. The assignment of various modes along the potential energy distribution (PED) is given in Table II. The assignments have been made on the basis of PED, absorption band shape, band intensity and absorption/scattering in 


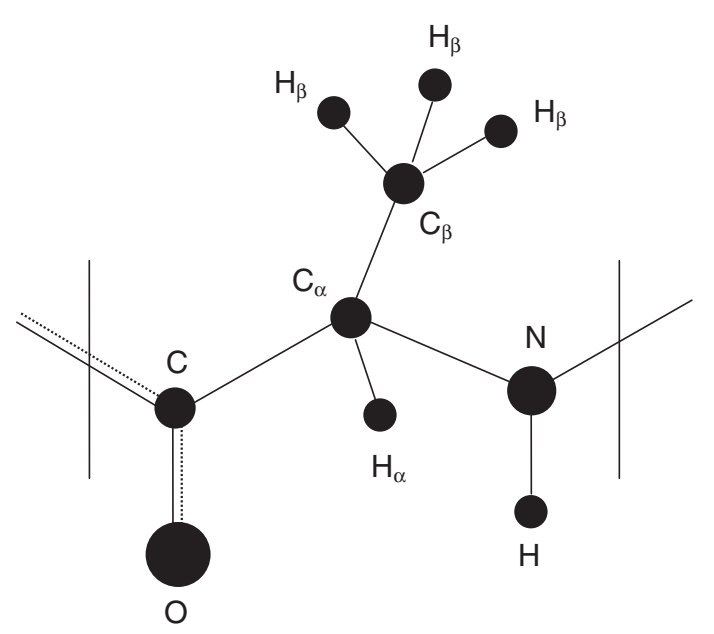

Figure 1. One chemical repeat unit of $\beta$ PLA.

similar molecules having groups placed in similar environments. For the sake of simplicity the modes are discussed under two heads, viz., backbone modes and side-chain modes.

\section{Backbone Modes}

The mode involving the motions of main chain atoms $(-\mathrm{CO}-\mathrm{C} \alpha-\mathrm{NH}-)$ are termed as backbone modes. Amide groups of polypeptides are strong absorber in IR region, and these groups give rise to strong characteristic bands (Amide A, I-VII). These amide modes along with other characteristic modes have been used for the structural diagnosis. Based on such diagnostic correlations, attempts have been made to estimate secondary structural composition in proteins. A comparison of the amide modes of $\beta$ PLA with the other polypeptides ${ }^{4,10,28}$ having $\beta$ sheet conformation is given in Table III.

The amide A mode which arises due to the $\mathrm{N}-\mathrm{H}$ stretch, is not too sensitive to chain conformation and side-chain structure, but is highly sensitive to the strength of the $\mathrm{N}-\mathrm{H} \cdots \mathrm{O}=\mathrm{C}$ hydrogen bond. As seen in Table III, amide A in $\beta$ PLA has the frequency which is lower than poly(L-serine) (PLS) and PG I. It should be noted that the value of the $\mathrm{N}-\mathrm{H}$ stretch force constant for $\beta$ PLA is lower than that for PG I. This is consistent with the stronger hydrogen bond in the pleated sheet structure as compared to the rippled-sheet. ${ }^{29,30}$

Amide I mode is calculated at $1671 \mathrm{~cm}^{-1}$ at $\delta=0$ and is assigned to the observed peak at $1669 \mathrm{~cm}^{-1}$ in Raman spectra. Amide II mode is calculated at $1536 \mathrm{~cm}^{-1}$ both at the zone centre and zone boundary and can be assigned to Raman band at $1538 \mathrm{~cm}^{-1}$. The $1555 \mathrm{~cm}^{-1}$ peak in Raman and $1524 \mathrm{~cm}^{-1}$ peak in polarized IR spectra arise from the crystal field splittings as discussed by Moore and Krimm. ${ }^{13}$ This mode lies in the same range in other polypeptides having $\beta$
Table III. Comparison of amide modes of $\beta$ PLA with other polypeptides in $\beta$ form

\begin{tabular}{lrrrrrrrrr}
\hline \multirow{2}{*}{ Amide } & \multicolumn{2}{c}{$\beta$ PLA } & \multicolumn{2}{c}{$\beta$ PLV } & \multicolumn{2}{c}{$\beta$ PG I } & \multicolumn{2}{c}{$\beta$ PLS } \\
\cline { 2 - 10 } & $\delta=0$ & $\delta=\pi$ & $\delta=0$ & $\delta=\pi$ & $\delta=0$ & $\delta=\pi$ & $\delta=0$ & $\delta=\pi$ \\
\hline A & 3242 & 3242 & 3290 & 3290 & 3398 & 3298 & 3318 & 3318 \\
I & 1669 & 1669 & 1638 & 1638 & 1650 & 1628 & 1637 & 1628 \\
II & 1538 & 1538 & 1545 & 1545 & 1520 & 1520 & 1532 & 1537 \\
III & 1223 & 1243 & 1228 & 1228 & 1300 & 1281 & 1249 & 1270 \\
IV & 528 & 698 & 548 & 684 & 628 & 711 & 533 & 773 \\
V & 622 & 709 & 715 & 715 & 705 & 725 & 713 & 685 \\
VI & 528 & 657 & 615 & 628 & 590 & 600 & 533 & 647 \\
\hline
\end{tabular}

All frequencies are in $\mathrm{cm}^{-1}$

PLA: poly(L-alanine) PLV: poly(L-valine $)^{4}$

PG I: poly(L-glycine $)^{10}$ PLS: poly(L-serine $)^{28}$

\section{(Table III) sheet conformation.}

The frequency of the amide III mode does not depend solely on main-chain conformation. Side-chain structure also plays an important role. ${ }^{31}$ The amide III mode calculated at $1224 \mathrm{~cm}^{-1}$ at the zone centre is assigned to the observed peak at $1221 / 1223 \mathrm{~cm}^{-1}$ in Raman/IR spectra. At the zone boundary it is calculated at $1236 \mathrm{~cm}^{-1}$ and matched to the observed band at $1241 / 1243 \mathrm{~cm}^{-1}$ Raman/IR spectra. This matching could be made only when the off-diagonal coupling interaction between $v(\mathrm{C}=\mathrm{O})$ and $\varphi(\mathrm{O}=\mathrm{C}=\mathrm{N})$ and $v(\mathrm{C}=\mathrm{N})$ and $\varphi(\mathrm{C}=\mathrm{N}-\mathrm{H})$ was taken into consideration. The motion of these internal coordinates becomes coupled via the motion of the carbon atom of the carbonyl group and via the Nitrogen atom of the peptide group.

The amide IV mode calculated at $528 \mathrm{~cm}^{-1}$ at $\delta=$ 0 and is assigned to the observed peak at the same value, is coupled mode mixed with amide VI. Krishnan et al. ${ }^{12}$ have assigned this mode at $594 \mathrm{~cm}^{-1}$. The peak at $594 \mathrm{~cm}^{-1}$ is not observed in the spectra of $\beta$ PLA but in IR spectra and INS of $\alpha$ PLA.

The $\mathrm{N}-\mathrm{H}$ wagging mode, which give rise to the amide $\mathrm{V}$ is calculated at $631 \mathrm{~cm}^{-1}$ at the zone centre and is assigned to the observed IR peak at 622 $\mathrm{cm}^{-1}$. Its frequency depends on a combination of main-chain conformation, hydrogen bond strength and side-chain structure. Although interchain interactions through hydrogen bonds are not explicitly used in the present calculations for the isolated chain, but its effects are indirectly taken into account in the search for the "best" force field for the neighbouring alanine residue.

Elliott ${ }^{32}$ speculated that the observed IR band at $1308 \mathrm{~cm}^{-1}$ in $\beta$ PLA was probably due to the presence of some $\alpha$-helical conformation in $\beta$ sample. Actually in the IR spectrum of $\beta$ PLA, there are two bands in this region, at 1332 and $1308 \mathrm{~cm}^{-1}$. From the spectrum it is not clear if these absorptions are in- 
deed spectral contributions from $\beta$ or are $\alpha$-helically linked alanine residues. However, in Raman spectrum of $\beta$ PLA the bands at 1335 and $1311 \mathrm{~cm}^{-1}$ have intensities which are too large relative to the intensities of the corresponding bands in the $\alpha$ form. Bands near these frequencies are predicted by our calculations as $\left(\mathrm{C}_{\alpha}-\mathrm{H}_{\alpha}\right)$ bending. The appearance of $\left(\mathrm{C}_{\alpha}-\mathrm{H}_{\alpha}\right)$ bending mode in $\mathrm{PLS}^{28}$ in the same range also supports our assignment.

\section{$\mathrm{CH}_{3}$ as a Dynamical Unit}

The introduction of $\mathrm{CH}_{3}$ as a dynamical unit brings about significant changes in the assignments and in the dispersive behaviour of normal modes lying below $1400 \mathrm{~cm}^{-1}$. They are shown in Table II. These are the modes, which belong either to the $\mathrm{CH}_{3}$ group or appear with the $\mathrm{CH}_{3}$ group as a mixture of several skeletal and non-skeletal modes. The $\mathrm{CH}_{3}$ symmetric stretch, asymmetric stretch and asymmetric deformation are pure modes. However the symmetric deformation does mix with its neighbouring angle bends $\left(\mathrm{C}_{\alpha}-\mathrm{C}_{\beta}-\mathrm{H}_{\beta}\right)$. These motions are electrically and mechanically coupled. The main dispersive modes are $\mathrm{CH}_{3}$ torsion and $\mathrm{CH}_{3}$ rock, which appear at the zone centre at 266 and $906 \mathrm{~cm}^{-1}$, respectively, and disperse to 300 and $926 \mathrm{~cm}^{-1}$ at the zone boundary. However, there are other modes which appear at 1060 and $981 \mathrm{~cm}^{-1}$ at $\delta=0$ and have significant contribution from the $\mathrm{CH}_{3}$ rock. As the $266 \mathrm{~cm}^{-1}$ mode progresses towards the zone boundary, its character starts changing and at $\delta=0.40 \pi$, it suffers repulsion with another mode which appears at $185 \mathrm{~cm}^{-1}$ at the zone centre. After repulsion there is an exchange of PE. Such points of repulsion/cross-over generally refer to some internal symmetry points in the energy momentum space. With $\mathrm{CH}_{3}$ as a dynamical unit some new mode have appeared which are not purely $\mathrm{CH}_{3}$ mode but are coupled with several other modes. The modes calculated at 1404 and $1330 \mathrm{~cm}^{-1}$ at $\delta=0$ belong to this category. These modes were not observed by Krishnan et al. ${ }^{12}$ The mode at $1404 \mathrm{~cm}^{-1}$ is a skeletal angle bend $\varphi\left(\mathrm{N}-\mathrm{C}_{\alpha}-\mathrm{H}_{\alpha}\right)$ and mixes with $\left(\mathrm{C}_{\alpha}-\mathrm{C}_{\beta}-\mathrm{H}_{\beta}\right)$ and $\left(\mathrm{H}_{\beta}-\mathrm{C}_{\beta}-\mathrm{H}_{\beta}\right)$. The other mode is the bending of $\left(\mathrm{C}_{\alpha}-\right.$ $\left.\mathrm{H}_{\alpha}\right)$ and it also mixes with $\left(\mathrm{C}_{\alpha}-\mathrm{C}_{\beta}-\mathrm{H}_{\beta}\right)$ and $\left(\mathrm{H}_{\beta}-\right.$ $\mathrm{C}_{\beta}-\mathrm{H}_{\beta}$ ). Since these modes are dynamically coupled, hence the profile of dispersion curves is also changed.

The introduction of $\mathrm{CH}_{3}$ as a dynamical unit leads to the revision of some of the assignments made earlier. ${ }^{12}$ They have assigned the peak at $1167 \mathrm{~cm}^{-1}$ due to angle bends $\varphi\left(\mathrm{C}-\mathrm{C}_{\alpha}-\mathrm{C}_{\beta}\right)$ and $\varphi\left(\mathrm{N}-\mathrm{C}_{\alpha}-\mathrm{C}_{\beta}\right)$. Now, on comparison with other polypeptides, this mode is assigned as skeletal stretching mode of $\left(\mathrm{N}-\mathrm{C}_{\alpha}\right)$ and $\left(\mathrm{C}-\mathrm{C}_{\alpha}\right)$. This mode is partially coupled with $\left(\mathrm{C}_{\alpha}-\right.$ $\mathrm{C}_{\beta}-\mathrm{H}_{\beta}$ ). Our assignment agrees with that made by Moore and Krimm. ${ }^{13}$ Also this mode appears in the same range in $\beta$ PLV. ${ }^{4}$ The $\tau(\mathrm{C}-\mathrm{N})$ which was assigned to $247 \mathrm{~cm}^{-1}$ at $\delta=0$ is incorrect and should be assigned to $91 \mathrm{~cm}^{-1}$ at $\delta=0$ and at $122 \mathrm{~cm}^{-1}$ at $\delta=\pi$. The mode calculated at $247 \mathrm{~cm}^{-1}$, which appears intense in INS at $230 \mathrm{~cm}^{-1}$, should be assigned to the methyl torsion. Its intensity in INS is due to large amplitude of motion.

\section{Dispersion Curves}

The dispersion of modes below $1350 \mathrm{~cm}^{-1}$ is plotted in Figures 2a and 3a. All modes above this are non dispersive. As shown in Figure $3 \mathrm{a}$ the two lowest lying branches ( $\nu=0$ at $\delta=0$ and $\delta=\pi$ ) are the four acoustic modes. At $\delta=0$ the two acoustic branches correspond to the translational mode along the chain axis and rotational mode about the axis. At $\delta=\pi$, they correspond to the translational modes perpendicular to the rotational axis.

The mode calculated at $1173 \mathrm{~cm}^{-1}$ at $\delta=0$ is assigned to the observed peak at $1167 / 1165 \mathrm{~cm}^{-1}$ in Raman/IR. It shows a dispersion of 81 wave number. This mode has a dominant contribution of skeletal stretches $\left[v\left(\mathrm{~N}-\mathrm{C}_{\alpha}\right)(44 \%)+v\left(\mathrm{C}_{\alpha}-\mathrm{C}\right)(17 \%)\right]$ at the zone centre. On moving away from the zone centre contribution of $v\left(\mathrm{~N}-\mathrm{C}_{\alpha}\right)$ and $v\left(\mathrm{C}_{\alpha}-\mathrm{C}\right)$ decreases and that of $v(\mathrm{C}-\mathrm{N})$ and $v\left(\mathrm{C}_{\alpha}-\mathrm{C}_{\beta}\right)$ increases. At the zone boundary, this mode reaches $1092 \mathrm{~cm}^{-1}$ and is assigned to the peak at $1096 \mathrm{~cm}^{-1}$ in Raman spectra.

The mode at $1060 \mathrm{~cm}^{-1}$ at the zone centre, assigned to the observed peak at 1050 (Raman) $\mathrm{cm}^{-1}$, shows a dispersion of 55 wave number. This mode has the dominant contribution of skeletal as well as side-chain stretches. The contribution of skeletal stretches decreases with $\delta$ and that of side-chain stretches and methyl bending increases. At zone boundary this mode reaches $1005 \mathrm{~cm}^{-1}$ and is assigned to the peak at $1017 \mathrm{~cm}^{-1}$ in IR spectra.

The mode calculated at $775 \mathrm{~cm}^{-1}$ at $\delta=0,[\omega(\mathrm{N}-$ $\mathrm{H})(37 \%)+\omega(\mathrm{C}=\mathrm{O})(26 \%)]$ is assigned to the observed peak at $770 \mathrm{~cm}^{-1}$. The $630 \mathrm{~cm}^{-1}$ mode at $\delta=0$, $[\omega(\mathrm{N}-\mathrm{H})(30 \%)+\tau(\mathrm{C}-\mathrm{N})(18 \%)+\omega(\mathrm{C}=\mathrm{O})(13 \%)]$ is Amide $\mathrm{V}$ which is assigned to the observed peak at $622 \mathrm{~cm}^{-1}$. The PED of these two modes shows no appreciable change up to $\delta=0.40 \pi$. Beyond this they start interacting and come closer. In the $775 \mathrm{~cm}^{-1}$ mode contribution of $\omega(\mathrm{N}-\mathrm{H})$ starts increasing while its frequency decreases with $\delta$ and reaches $702 \mathrm{~cm}^{-1}$ at $\delta=\pi$, where it is assigned to Amide $\mathrm{V}$ mode and corresponds to the observed peak at $709 \mathrm{~cm}^{-1}$ in IR spectra. On the other hand, the contribution of the $\mathrm{C}=\mathrm{O}$ in-plane bending increases in the mode at $630 \mathrm{~cm}^{-1}$. This mode reaches $698 \mathrm{~cm}^{-1}$ at $\delta=\pi$ and becomes Amide IV mode.

The mode at $528 \mathrm{~cm}^{-1}$ at zone centre has the major contribution from the $\mathrm{C}=\mathrm{O}$ in-plane bending along 


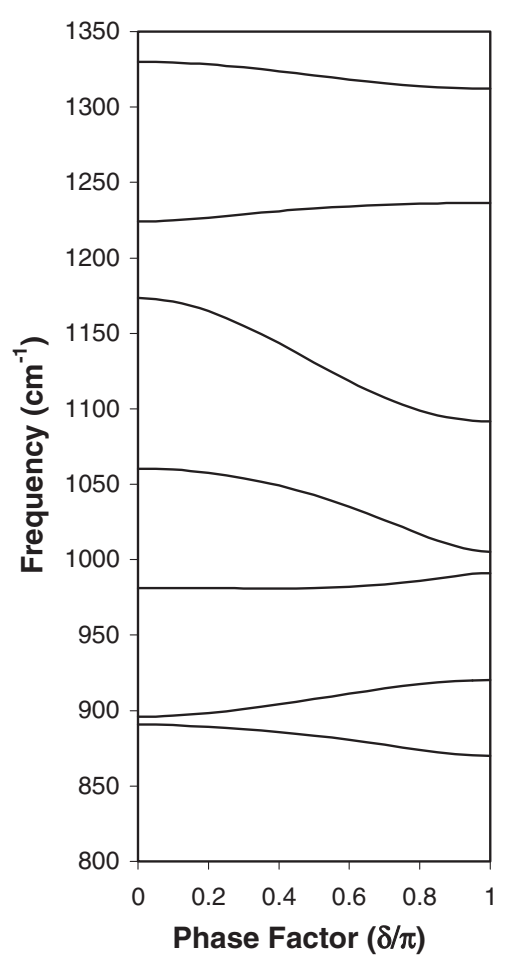

(a)

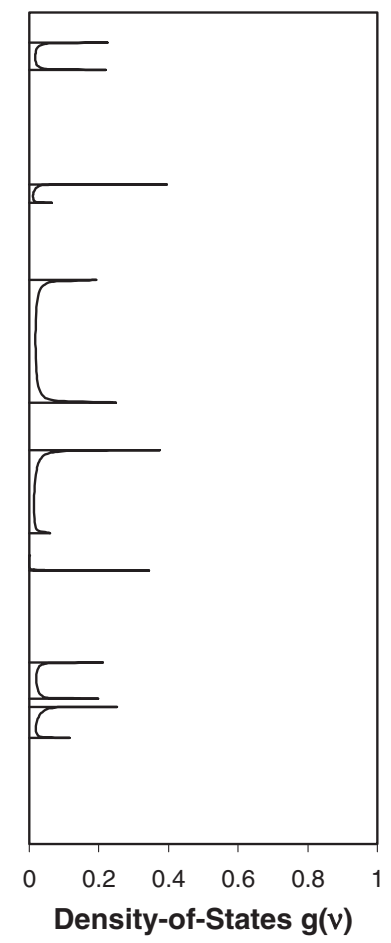

(b)

Figure 2. (a) Dispersion curves and (b) density of states of $\beta$ PLA $\left(1350-800 \mathrm{~cm}^{-1}\right)$.

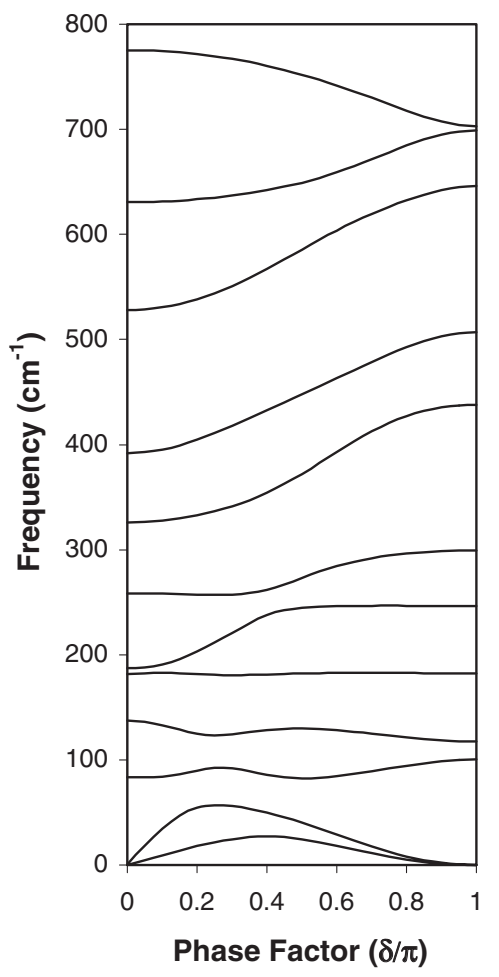

(a)

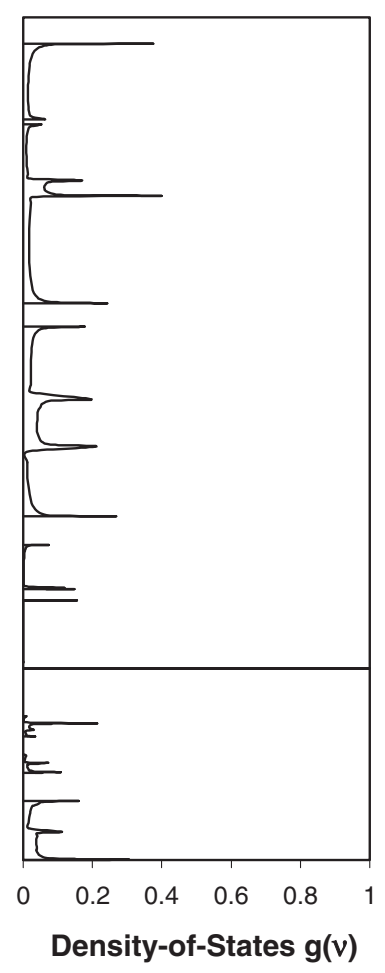

(b)

Figure 3. (a) Dispersion curves and (b) density of states of $\beta$ PLA below $800 \mathrm{~cm}^{-1}$.

with it's out-of-plane bending $\left[\varphi\left(\mathrm{C}_{\alpha}-\mathrm{C}=\mathrm{O}\right)(29)+\right.$ $\varphi(\mathrm{O}=\mathrm{C}-\mathrm{N})(24)+\omega(\mathrm{C}=\mathrm{O})(20)]$. It is assigned to the observed peak at the same frequency. The contribution of $(\mathrm{C}=\mathrm{O})$ in plane bending decreases and that of the $\mathrm{C}=\mathrm{O}$ out-of-plane bending increases in this mode with increasing $\delta$. At $\delta=\pi$. it reaches 646 $\mathrm{cm}^{-1}\left[\omega(\mathrm{C}=\mathrm{O})(50 \%)+\tau\left(\mathrm{N}-\mathrm{C}_{\alpha}\right)(12 \%)\right]$ and is assigned to the observed peak at $657 \mathrm{~cm}^{-1}$ (Amide VI). 


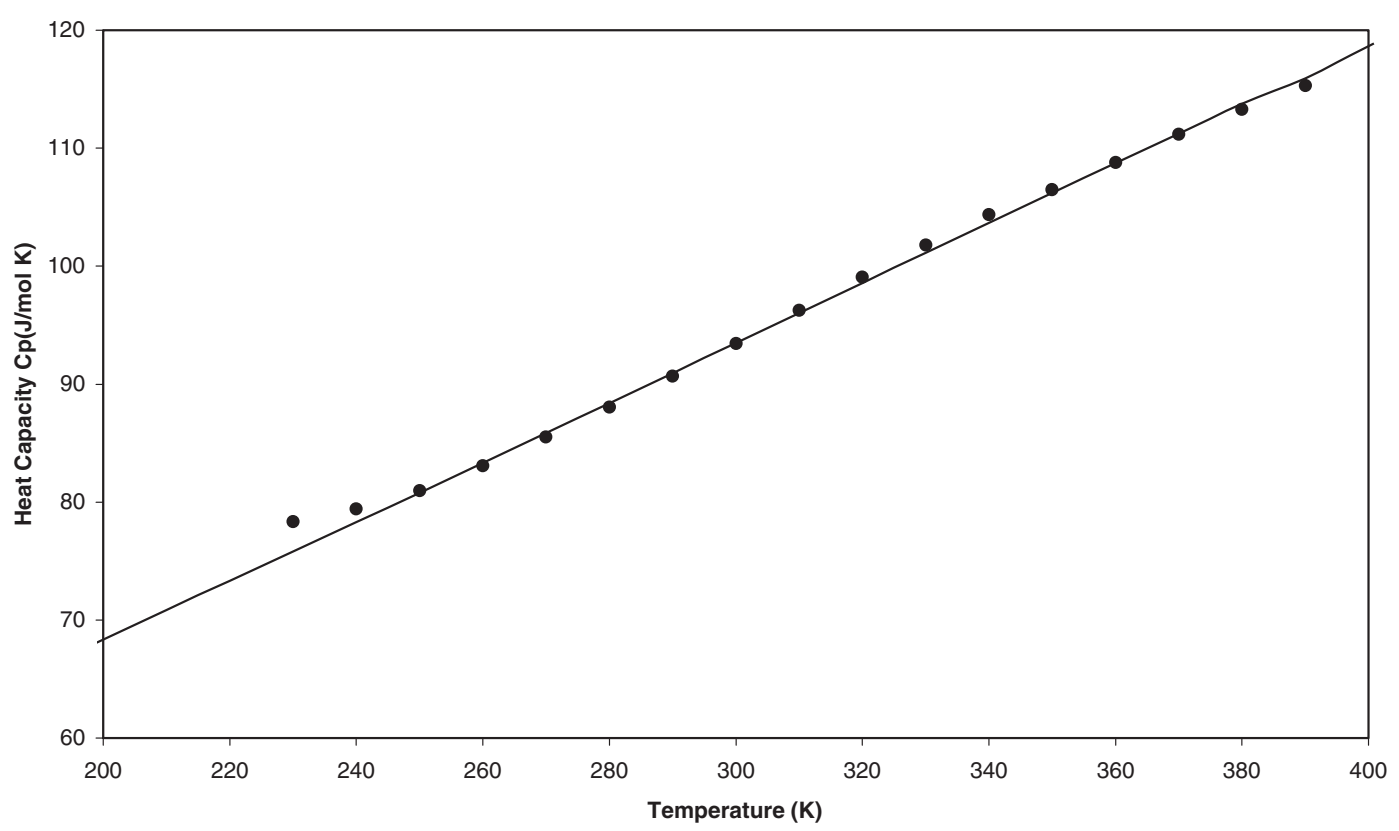

Figure 4. Variation of heat capacity of $\beta$ PLA as a function of temperature [theoretical values (一) and experimental data (๑)].

The modes calculated at 392 and $326 \mathrm{~cm}^{-1}$, which arises due to skeletal angle bending modes, are highly dispersive because of strong coupling along the chain.

One of the characteristic features of the dispersion curves is the repulsion and exchange of character between two pairs of modes. Pairs of modes that repel appear at 259 and $187 \mathrm{~cm}^{-1}$ at the zone centre. The repulsion occurs in the neighbourhood of $0.40 \pi$. It is observed that at this $\delta$ value, the modes come close, exchange their PED and then repel. After repulsion, the second mode becomes non dispersive, while the first one disperses progressively with $\delta$. This interesting phenomenon of exchange of character may be viewed as the collision of two phonons approaching to each other and moving apart after exchanging their PED. Such repulsion occurs for modes belonging to same symmetry species. ${ }^{33}$

The dispersion curves in the lower frequency region $\left(150-400 \mathrm{~cm}^{-1}\right)$ fan out from the zone centre towards the zone boundary, appearing to repel each other. This feature is also true of the dispersion curves of PG I, ${ }^{10}$ $\beta$ PLS, ${ }^{28}$ and polyethylene (PE) ${ }^{34}$ which take planar zig-zag configuration. This common feature may be due to the absence of strong interchain interactions, stabilizing the structure, in contrast to the case of the hydrogen-bonded $\alpha$-helix.

An interesting feature in the dispersion curves is the parallelism between the 528, 392 and $326 \mathrm{~cm}^{-1}$ modes. Since the phonon velocity is related to the gradient of the dispersion curve, it shows same velocity for the three phonons.

The lower frequency modes, especially the acoustic modes, are the characteristic of 2-fold helical chain symmetry. The two acoustic branches in the disper- sion curves are similar in shape to the dispersion of these branches in other 2-fold helical polymers like $\mathrm{PE},{ }^{34} \mathrm{PG} \mathrm{I},{ }^{10} \beta \mathrm{PLV}^{4}$ and syndiotactic polystyrene in its two-fold helical form, ${ }^{35}$ etc. The peaks in the acoustic curves of $\beta$ PLA occur at $\delta=0.45 \pi$ and $\delta=0.25 \pi$. A comparison with $\beta$ PLV shows that, although the peaks in the acoustic curves fall at about the same $\delta$ value, the peaks heights are different due to different side-chain structure.

Agreement with the experimental values for frequencies at $\delta=0$ and $\delta=\pi$ shows that basically the profile of dispersion curves is correct.

\section{Density-of-States and Heat Capacity}

Frequency distribution function as obtained from the dispersion curves is shown in Figures $2 b$ and $3 b$. The peaks in the frequency distribution curves correspond to the regions of high density-of-states. The observed frequencies correspond well with these peak positions. From this knowledge of density-of-states heat capacity of $\beta$ PLA chain has been calculated in the range 200-400 K (Figure 4). In the figure the solid circles represent experimental data of Roles and Wunderlich $^{27}$ Our calculations agree well with the experimental data except at the low temperatures.

The discrepancy at the low temperature could arise for two reasons, one the neglect of interchain interactions at low temperature and the other because of force field being temperature independent. The former leads to low frequency lattice modes, which are not included in the present model, and the heat capacity is sensitive to it. The evaluation of lattice modes is not only prohibitive dimensionally but even the enormity of interactions, which are difficult to visualize. 
As for the latter, the temperature dependence of force field is difficult to build in the potential field. Such measurement and evaluation of heat capacity can give information about the portion of protein which is in $\alpha$ helical or $\beta$ sheet structure and is necessary in evaluating the basic thermodynamics of enzyme reaction.

\section{CONCLUSIONS}

The use of $\mathrm{CH}_{3}$ as a dynamical unit rather than as a mass point has emerged in correcting several assignments both at the zone centre and at the zone boundary. Further, the change in the dispersion profile results in good agreement with the heat capacity measurements on $\beta$ PLA.

Acknowledgment. Financial assistance to one of the authors (PT) from the Department of Science and Technology, New Delhi under Fast Track Scheme for Young Scientists, is gratefully acknowledged.

\section{REFERENCES}

1. K. J. M. Das, S. Rastogi, P. Tandon, and V. D. Gupta, Eur. Polym. J., 37, 2295 (2001).

2. D. Kapoor, N. K. Misra, P. Tandon, and V. D. Gupta, J. Macromol. Sci., Part B: Phys., 37, 723 (1998).

3. E. Henssge, D. Dumont, D. Fischer, and D. Bougeard, J. Mol. Struct., 482-483, 491 (1999).

4. L. Burman, P. Tandon, V. D. Gupta, and S. Srivastava, Biopolymers, 38, 53 (1996).

5. A. Elliott and B. R. Malcolm, Proc. R. Soc. London, Ser. A, 249, 30 (1959).

6. S. Arnott, S. D. Dover, and A. J. Elliott, Mol. Biol., 30, 201 (1967).

7. S. Pande, P. Tandon, and V. D. Gupta, J. Macromol. Sci., Part B: Phys., 41, 1 (2002).

8. P. Sharma, N. K. Misra, P. Tandon, and V. D. Gupta, J. Macromol. Sci., Part B: Phys., 41, 319 (2002).

9. S. Pande, P. Tandon, and V. D. Gupta, J. Polym. Sci., Part B: Polym. Phys., 42, 316 (2004).

10. V. Porval, R. M. Misra, P. Tandon, and V. D. Gupta, Indian J. Biochem. Biophys., 41, 34 (2004).
11. V. D. Gupta, S. Trevino, and H. Boutin, J. Chem. Phys., 48, 3008 (1968).

12. M. V. Krishnan and V. D. Gupta, Indian J. Pure Appl. Phys., 10, 210 (1972).

13. W. H. Moore and S. Krimm, Biopolymers, 15, 2465 (1976).

14. W. Drexel and W. L. Peticolas, Biopolymers, 14, 715 (1975).

15. K. Datye Vandana and S. Krimm, J. Chem. Phys., 84, 6989 (1986).

16. E. B. Wilson, J. C. Decius, and P. C. Cross, "Molecular Vibrations: The Theory of Infrared and Raman Vibrational Spectra,” Dover Publications, Mineola, N.Y., 1980.

17. P. W. Higgs, Proc. R. Soc. London, Ser. A, 220, 472 (1953).

18. B. Mannfors, K. Palmo, and S. Krimm, J. Mol. Struct., 556, 1 (2000).

19. H. C. Urey and C. A. Bradley, Phys. Rev., 38, 1969 (1931).

20. W. Qian, N. G. Mirikin, and S. Krimm, Chem. Phys. Lett., 315, 125 (1999).

21. K. Itoh, T. Nakahara, and T. Shimanouchi, Biopolymers, 6, 1759 (1968).

22. K. Itoh and H. Katabuchi, Biopolymers, 11, 1593 (1972).

23. B. G. Frushor and J. L. Koenig, Biopolymers, 13, 455 (1974).

24. B. Franconi, Biopolymers, 11, 1817 (1973).

25. W. T. King, I. M. Mills, and B. L. Crawford, J. Chem. Phys., 27, 455 (1957).

26. R. Pan, M. Verma-Nair, and B. Wunderlich, J. Therm. Anal., 35, 955 (1989).

27. K. A. Roles and B. Wunderlich, Biopolymers, 31, 477 (1991).

28. A. Gupta, P. Tandon, V. D. Gupta, and S. Rastogi, Polymer, 38, 2389 (1997).

29. L. Pauling and R. B. Corey, Proc. Natl. Acad. Sci. U.S.A., 39, 253 (1953).

30. B. Lotz, J. Mol. Biol., 87, 168 (1974).

31. S. Krimm and J. Bandekar, Adv. Protein Chem., 38, 181 (1986).

32. A. Elliott, Proc. R. Soc. London, Ser. A, 226, 408 (1954).

33. D. I. Bower and W. F. Maddams, "The Vibrational Spectroscopy of Polymers," Cambridge University Press, New York, N.Y., 1989, p 154.

34. M. Tasumi and T. Shimanouchi, J. Mol. Spectrosc., 9, 261 (1962).

35. S. Rastogi and V. D. Gupta, J. Macromol. Sci., Part B: Phys., 34, 1 (1995). 